\title{
Cardiac Pacemaker and Wireless Capsule Endoscopy Interference: Case Report in a Patient with Gastric Vascular Ectasias
}

\author{
A. G. Gravina ${ }^{1}$, R. Bozzi ${ }^{2}$, I. J. Romano ${ }^{3}$, E. Pezzullo ${ }^{3}$, A. Miranda ${ }^{1}$, M. G. Merola ${ }^{1}$, \\ M. Romano ${ }^{1}$, A. Pezzullo ${ }^{1}$ \\ ${ }^{1}$ Dipartimento Medico Chirurgico di Internistica Clinica e Sperimentale, Gastroenterologia e C.I.R.A.N.A.D., \\ Seconda Università degli Studi di Napoli, Napoli, Italy \\ ${ }^{2}$ U.O.C. Chirurgia ed Endoscopia Digestiva e, Napoli, Italy \\ ${ }^{3}$ Cardiologia A.O.R.N. V. Monaldi, Napoli, Italy \\ E-mail:marco.romano@unina2.it \\ Received December 29, 2009; revised January 13, 2010; accepted January 17, 2010
}

\begin{abstract}
Wireless capsule endoscopy is a new endoscopic tool for the diagnosis and management of small bowel diseases. The main indication at present is the evaluation of GI bleeding of obscure origin, Crohn's disease, coeliac disease and small bowel tumors. Studies suggest that capsule endoscopy is associated with few adverse events. Whether cardiac pacemaker may interfere with capsule endoscopy is still a controversial issue. We here report a case showing that there is a possibility of interference between the two procedures, cardiac pacemaker affecting the proper functioning of capsule endoscopy and that this is related to the distance between the pacemaker and the recorder.
\end{abstract}

Keywords: Capsule Endoscopy, Cardiac Pacemaker, Interference, Vascular Ectasias

\section{Summary}

Wireless capsule endoscopy is a new endoscopic tool for the diagnosis and management of small bowel diseases. The main indication at present is the evaluation of GI bleeding of obscure origin, Crohn's disease, coeliac disease and small bowel tumors. Studies suggest that capsule endoscopy is associated with few adverse events. Whether cardiac pacemaker may interfere with capsule endoscopy is still a controversial issue. We here report a case showing that there is a possibility of interference between the two procedures, cardiac pacemaker affecting the proper functioning of capsule endoscopy and that this is related to the distance between the pacemaker and the recorder.

\section{Case Report}

A 75 year old man with ischemic cardiopathy with dilatative evolution treated with aorto-coronaric bypass, complicated by cardiac cirrhosis and mild ascites and renal failure came to our observation because of melena. He had been implanted a cardiac pacemaker because of a complete atrioventricular block (pacemaker model Biotronik-Kalos 05 VVI n/s 36012460 with electrode Biotronik HL 150, n/s 56756B). Conventional diagnostic interventions, including upper and lower endoscopy, failed to identify the site of bleeding. An enteroclysis was performed and resulted normal. The patient underwent a small bowel study by wireless capsule endoscopy (Endocapsule Olympus $\left.{ }^{\circledR}\right)$. The recorder was positioned ne$\mathrm{xt}$ to external abdominal sites near the pulse generator while the electrocardiogram was continuously recorded and reviewed by a cardiologist, to confirm the absence of pacing inhibition by the video transmission. During videocapsule examination, we revealed an interference lasting 20 minutes (Figure 1), with complete absence of any image on the real time viewer. The interference with videocapsule proper recording was subsequently confirmed after downloading on the workstation. In particular, we found this interference only when the recorder was positioned near the cardiac pacemaker while no interferences were found when the recorder was positioned at a distance from the pacemaker. The patient had continuous cardiac monitoring for the duration of the procedure and no arrhythmias or other adverse cardiac events 
were noted during videocapsule endoscopy. Wireless capsule endoscopy revealed the presence of two vascular lesions in the gastric antrum (Figure 2), suggestive for gastric vascular ectasias. This was confirmed by an EGDS, performed on the same day (Figure 3).

\section{Discussions}

There is a theorical risk of electromagnetic interference between the UHF digital radiofrequency of the wireless videocapsule $(433.8 \mathrm{MHz})$, and mobile phones, implanted cardiac pacemaker or defibrillator devices. However, interference between wireless capsule endoscopy and cardiac pacemaker has been observed only in one case [1], but has not been confirmed by others [2-5].

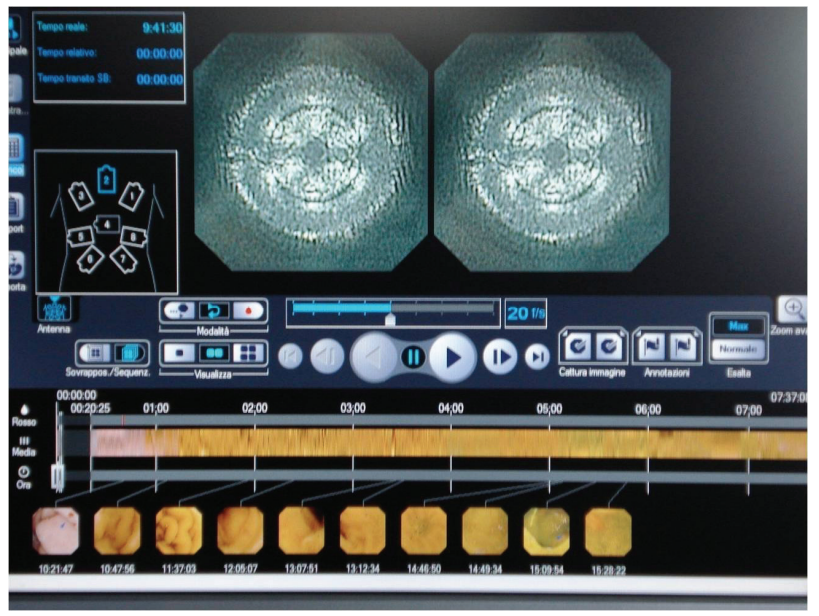

Figure 1. Workstation screen showing significant interference in the capsule recording.

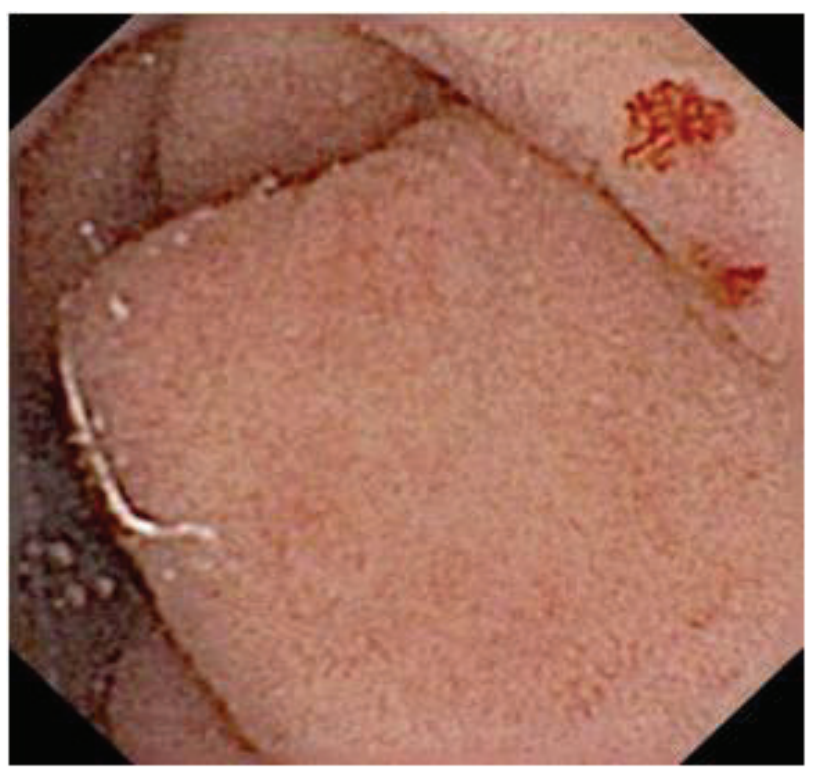

Figure 2. Wireless videocapsule imaging of the stomach showing two gastric lesions suggestive of vascular ectasias.

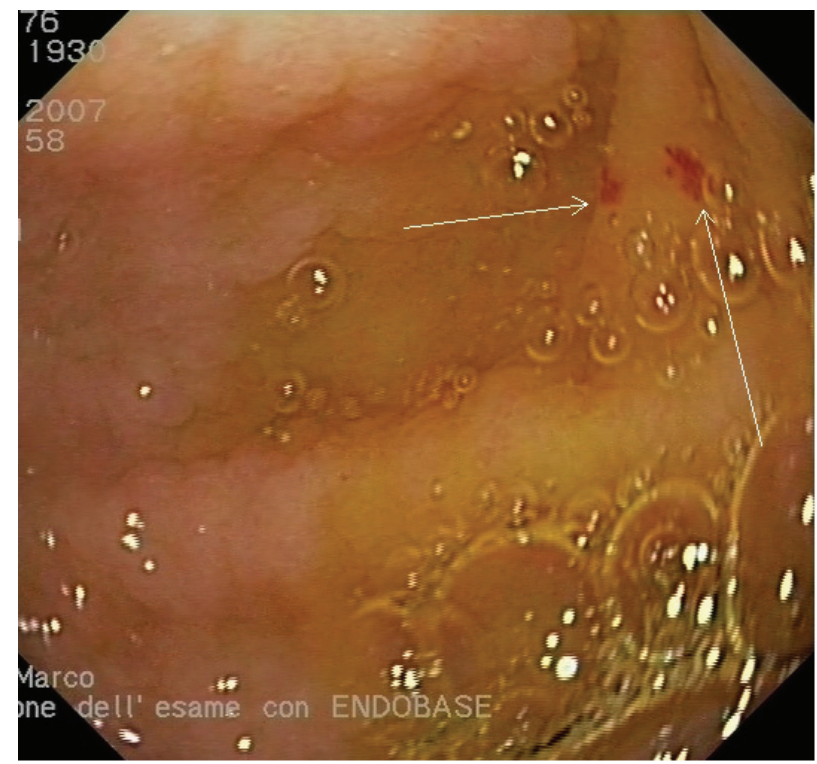

Figure 3. Esophagogastroduodenoscopy showing two vascular ectasias in the gastric antrum.

In our case, which is the first described using Endocapsule Olympus ${ }^{\circledR}$, we show that cardiac pacemaker may interfere with videocapsule proper functioning leading in our case to a "blank" period of approximately 20 minutes and this depends upon the distance between the recorder and the cardiac pacemaker. In particular no interferences were observed when a distance of about $20 \mathrm{~cm}$ between the two devices was maintained. Also, we confirm that wireless capsule endoscopy does not interfere with the correct function of cardiac pacemaker as assessed by continuous cardiac monitoring of the patient. Interestingly, wireless capsule endoscopy helped identify the gastric source of the bleeding then confirmed by a subsequent EGDS.

\section{References}

[1] Y. Guyomar, L. Vandeville, S. Heuls, et al., "Interference between pacemaker and video capsule endoscopy," Pacing and Clinical Electrophysiology, Vol. 27, pp. 1329 1330, 2004

[2] J. A. Leighton, V. K. Sharma, K. Srivathsan, et al., "Safety of capsule endoscopy in patients with pacemakers," Gastrointestinal Endoscopy, Vol. 59, pp. 567, 2004.

[3] G. Payeras, J. Piquersas, V. J. Morena, et al., "Effects of capsule endoscopy on cardiac pacemakers," Endoscopy, Vol. 37, pp. 1181-1185, 2005.

[4] M. H. Dirks, F. Costea, and E. G. Seidman, "Successful videocapsule endoscopy in patients with an abdominal cardiac pacemaker," Endoscopy, Vol. 40, pp. 73-75, 2008.

[5] D. Bandorski, W. Irnich, M. Bruck, et al., "Capsule endoscopy and cardiac pacemaker: Investigation for possible interference," Endoscopy, Vol. 40, pp. 36-39, 2008. 